Reprinted from September 1975, Vol. 97, Journal of Fluids Engineering

\section{Steady-State Flow in a Porous Cylinder With Permeable Walls and Restricted or Unrestricted Ends}

\section{P. Somasundaran' and K. J. Mysels ${ }^{2}$}

Even though flow through porous media has received considerable attention, flow through a medium bound by permeable boundaries has not been studied in any detail in the past. Media bound by permeable boundaries are however often encountered in practice. Flow of gases through a cigarette where both the tobacco rod and the wrapping paper are porous is of this type [1]. A closely related system is that of the graphite block in a gas-cooled nuclear reactor where the nuclear fuel is separated from the rapidly circulating coolant by a porous graphite septum with a narrow gap between fuel and septum [2].

Related problems having different geometries include seepage from streams bounded by porous banks [3], leakage into some aquifers [4] and the displacement of oil from sandstones by shalewater influx [5]. This type of flow presents certain peculiarities, such as the lack of simple additivity of effective resistances placed in series.

This paper presents a theory relating the significant waterial properties to the steady-state flow and pressure distribution and effective resistance for a uniform cylindrical system of the above type, first alone and then when coupled with resistances at either end. We shall consider the case in which one end of the

TRemench Department, R. J. Roynolds Industries, Winoton-Balem, N.C.; prently, Henry Krumb School of Mines, Columbia University, New York. N.Y.

Research Department, R. J. Reynolds Industrie, Winaton-Salem, N.C.; presently, 8327 La Jolla Scenic Drive, La Jolla, Celif.

'Numbers in bracketo deaignato References et ead of Brief.

Contributed by the Fluids Engineering Division of The Amencas Bocietr of Mechanical Evolvesse. Manubeript received at ASME Headquartors, June 3, 1975.

Journal of Fluids Engineering specimen is connected to a sink and the other, as well as the shell, to surroundings of uniform pressure. This is the cigarette case and differs in details from that of the reactor or the river, but shows the general features common to all such systems. The method used is similar to that employed in the treatment of flow of current through electric transmission lines [6] and in some ways also of heat transfer from fins [7].

Fig. 1 shows the essential elements of our system, a cylindrical core having a length $L$ and a linear resistance, $r_{e}$, to fow along it, surrounded by a shell having a linear resistance, $r_{\text {, }}$, to flow across it. The inlet of the specimen may carry a resistance $R_{i}$, the sink end another, $R_{\text {. }}$. The inlet and the whole shell are exposed to an extended source of constant pressure, which we take as the reference point, and the sink to a negative pressure $P(s)$. The flow at the sink is $F(s)$. By definition, the effective resistance of the system, $R$, is $-P(s) / F(s)$. To find the relations between these latter quantities, as well as the flow $F(x)$ and the pressure $P(x)$ at any point along the cylinder and its structural characteristics we make the foilowing assumptions:

1 the core and shell are homogeneous so that $r_{\text {s }}$ and $r_{6}$ are constant,

2 the flow across the core and that along the shell are both negligible,

3 flow is Darcian, and

4 the fluid is incompressible.

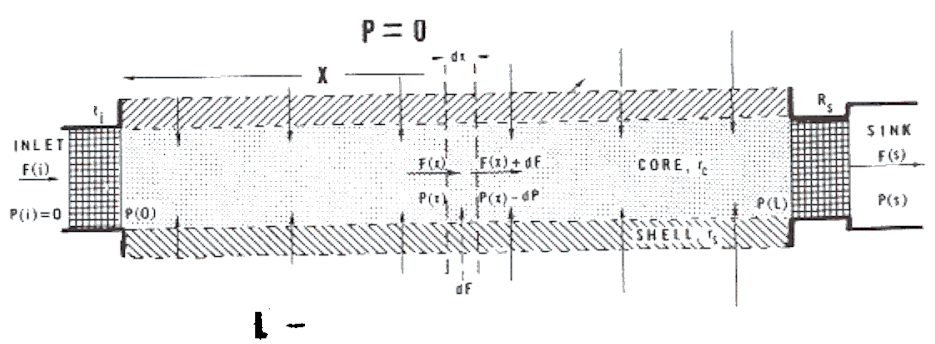

Fig. 1 
$x$ is the distance along the cylinder measured from the inlet. Within any small distance $d x$, the flow rate in the core changes then by $d F(x)$, which is the amount of fluid leaking in through the shell. This amount $d F(x)$ and the corresponding pressure drop $d P(x)$ are given by

$$
\begin{gathered}
d F(x)=-\left(P(x) / r_{0}\right) d x \\
-d P(x)=F r_{e} d x .
\end{gathered}
$$

The general solution of these differential equations is

$$
\begin{aligned}
& P=C_{1} \cosh \alpha x+C_{2} \sinh \alpha x \\
& F=C_{2} \cosh \alpha x+C_{4} \sinh \alpha x
\end{aligned}
$$

where the several $C$ 's are constants depending on boundary conditions, and $\alpha$ defined by

$$
\alpha=\left(r_{t} / r_{t}\right)^{1 / 2}
$$

is the natural unit of length in the system.

Uarestricted System. In this case $C_{1}=C_{4}=0$. We can then express the flow and pressure in terms of their values at the inlet or sink. Thus,

$$
\begin{aligned}
& P(x)=\frac{P(s) \sinh \alpha x}{\sinh \alpha L} \\
& F^{\prime}(x)=\frac{F(s) \cosh \alpha x}{\cosh \alpha L}
\end{aligned}
$$

The effective resistance of the system is given by

$$
R=\frac{-P(s)}{F(s)}=r_{k} \tanh \alpha L
$$

$r_{k}$, defined as

$$
r_{k}=\left(r_{e} r_{0}\right)^{1 / 2}
$$

is the resistance of an infinitely long system in which, necessarily, all the fluid enters through the shell. $r_{k}$ can be called the characteristic resistance of the system while $\alpha$ can be called the attenuation constant of the system. The complicated additivity of effective resistances of systems placed in series, is apparent.

Resistance at Inlet. Replacing resistance $R_{i}$ at the inlet by an extension of the system will give the same results for the initial core-and-shell provided that the resistance of this extension equals $R_{i}$. The required length of the extension, $\lambda$, can be obtained from equation (8) as

$$
\lambda=(1 / \alpha) \operatorname{arctanh}\left(R_{i} / r_{k}\right)
$$

When $R_{i}$ is larger than $r_{k}$, i.e., than the resistance of the infinitely long unrestricted system, $\lambda$ becomes imaginary. This complication can be avoided by using then the coth instead of the tanh in the definition of $\lambda$. In the extended system the total length is $L+\lambda$ and the coordinate of point $x$ becomes $x+\lambda$. Hence, for example, the flow at $x$ in terms of the flow at the sink end is given as

$$
F(x)=F(s) \frac{\left\{\begin{array}{c}
\cosh \\
\sinh
\end{array}\right\}(\alpha(x+\lambda))}{\left\{\begin{array}{c}
\cosh \\
\sinh
\end{array}\right\}(\alpha(L+\lambda))} \quad \text { for }\left\{\begin{array}{l}
R_{i} \leqslant r_{k} \\
R_{i} \geqslant r_{k}
\end{array}\right\}
$$

The effective resistance of the system is now

$$
R=r_{k}\left\{\begin{array}{c}
\tanh \\
\operatorname{coth}
\end{array}\right\} \alpha(L+\lambda) \quad \text { for }\left\{\begin{array}{l}
R_{i} \leqslant r_{k} \\
R_{i} \geqslant r_{k}
\end{array}\right\}
$$

which can be rewritten as

$$
R=\left(R_{i}+R_{m}\right) \frac{r_{z^{2}}}{r_{k}^{2}+R_{i} R_{w}}
$$

where $R_{*}$ is the resistance that the system would have if it were unrestricted, i.e., if $R_{i}$ were zero.

Equation (13) is the general expression for the addition of two systems in series. It should be noted that this addition is iterative, but not commutstive.

Two values of $R_{i}$ are of special interest; one is $r_{k}$, the other is infinity ( $R_{i}=0$ is of course the case of the unrestricted system discussed initially). When $R_{i}=r_{k}$

$$
\begin{aligned}
& F(x)=F(0) e^{a x} \\
& P(x)=P(0) e^{e x} .
\end{aligned}
$$

The resistance of this system is independent of its length. When $R_{i}=\infty$, i.e., when the inlet is closed, the value of $\lambda$ vanishes and the equations reduce to those for the unrestricted system with the sinh's replaced by cosh's, etc. This means that in the closedoff system, flows and pressures are completely interchanged as compared with the unrestricted system.

Resistance at Sink End. A simple leak-free resistance $R$, at the sink end makes the pressure at the sink no longer equal to that at $x=L$. Hence, the equations obtained in the preceding section are modified by replacing the sink pressure $P(s)$ by $P(0)$ and a similar set of results can be derived straightforwardly.

\section{References}

1 Mayer-Abich, K. M., Tobak Forschung 3:5, 1966, p. 367. 2 Ozisik, M. N. and Silverman, M. D. Trans. Am. Nucl. Soc. 3, 1970, p. 586; Nuclear Technology 14, 1972, p. 240; 16, 1972 , p. 577.

3 Bear, J., Zaslarsky, D., and Irmay, S. "Physical Principles of Water Percolation and Seepare," UNESCO, Paris, 1968 Polubarinova, Kochina, "Theory of Ground Water Movement," translated by J. M. Roger Do Wiest, Princeton University Press, Princeton, N. J., 1962; Wicke, E., and Brotz, W., Chem. Eng. Tech. 24, 1952, p. 58

4 Jacob, C. E., Trans. A m. Geophys. Union 27, 1946, p. 198; Hantush, M. S., and Jacob, C. E., ibid 36,1955 , p. 95.

5 Bobek. J. E., and Bail, P. T., Journal of Petroleum Technology, Sept. 1961, p. 950.

6 Kennelly, A. E., Electric Lines and Nets, McGraw-Hill, New York, 1928.

7 Eckert, E. R. G., and Drake, R. M., Jr., Heat and Mass Transfer, McGraw-Hill, New York, 1959, pp. 38-46. 